\title{
Corrigendum
}

The last page of the paper below was omitted by the printer from Vol. 12, Nos. 2/3 of Structural Optimization. Springer-Verlag has decided to publish the complete paper again on extra pages.

\section{Parametrical optimization of compression helical springs against instability}

\section{J. Krużelecki}

Institute of Mechanics and Machine Design, Cracow University of Technology, ul. Warszawska 24, 31-155 Kraków, Poland

\begin{abstract}
In the present paper, the parametrical optimization problem of helical springs against instability is considered. Such an initial distribution of a helix angle and radius of a spring is sought in the assumed class of functions which ensure maximal value of the critical axial compressive force under equality and inequality constraints. The volume of the spring material, the initial compression rigidity and also the slenderness ratio of the spring are considered as the equality constraints. The inequality constraints are connected with: a strength condition, a condition of closing up of neighbouring coils, and geometrical conditions in the initial and compressed state.
\end{abstract}

\section{Introduction}

The optimal design of helical springs is over 100 years old (Henderson 1884-1885), but the number of papers as well as variety of problems presented on this topic is rather scarce. Only the simplest parametrical optimization problems are considered; the optimal overall dimensions of a cylindrical spring with a constant pitch (for simplicity a helix angle is assumed to be zero) and/or maximal loadings are looked for and the applied constraints are not connected with buckling. A survey on the optimal design of helical springs is given by Kruzelecki (1990).

Optimization of compression helical springs with respect to their stability was initiated by Krużelecki (1993, 1995, 1996). Using the Pontryagin maximum principle the optimal distribution of a helix angle $\alpha_{0}(x)$ and radius $R_{0}(x)$ of a spring was looked for.

The present paper is continuation of problems presented by Krużelecki (1995), namely parametrical optimization of compression helical springs against instability is dealt with. Parametrical optimization means here that in the assumed functions, which describe the geometry of a spring (a helix angle, a radius of a spring), some free parameters are subjected to optimization. We look for such a value of parameters, distinguishing the geometry of a spring among a certain class of springs, which ensures the maximal value of a critical force. Utilization of the parametrical optimization can be justified by technological reasons, namely the manufacture of an optimal structure in the parametrical sense can be much simpler.

The formulation of the optimization problem in this paper, in general, is the same as discussed by Kruzelecki (1995). Therefore, we do not discuss it in detail. However, to make this paper easier to understand only a brief recapitulation is presented here and the differences and most important prob- lems are pointed out.

\section{Formulation of the optimization problem - state equations}

We consider a helical spring simply supported at both ends and loaded by an axial compressive force. We look for such an initial geometry of a spring (parameters in the functions which describe the geometry of a spring) with a given initial length $H_{0}$ which ensures the maximal critical force

$P_{\text {cr }} \rightarrow \max$,

under equality and inequality constraints. To calculate a critical force, the concept of an equivalent column proposed by Kruzelecki and Życzkowski (1990) is applied. A nonlinear precritical state with changes of geometry of a spring as well as with shearing effect according to the, so-called, modified approach discussed by Ziegler (1982), is taken into account. The appropriate differential equations of buckling for a simply supported spring-equivalent column in the dimensionless form can be written as follows:

$\frac{\mathrm{d} w}{\mathrm{~d} \xi}=\theta\left[\frac{\sin \alpha}{\sin \alpha_{0}}+\frac{p}{\mathrm{~d}^{4}} r^{2}(\xi) \frac{1+\nu \sin ^{2} \alpha}{2 \sin \alpha_{0}} \frac{1}{\mu_{s}}\right]$,

$\frac{\mathrm{d} \theta}{\mathrm{d} \xi}=-w \frac{p}{\mathrm{~d}^{4}} \frac{2+\nu \cos ^{2} \alpha}{2 \sin \alpha_{0}}$,

where $w=\bar{w} / H_{0}$ is a latteral displacement, $\xi=X / H_{0}$ a length variable $(0 \leq \xi \leq 1)$, $\theta$ the inclination of a normal to the cross-section against the central axis of an undeformed column, $r(\xi)=R(\xi) / H_{0}, \mu_{s}$ depends on an actual number of coils $n$, namely:

$\mu_{s}=\max \begin{cases}1-0.3576 / n, & n \geq 0.7152, \\ 0.5, & n \leq 0.7152,\end{cases}$

and was discussed by Krużelecki and Życzkowski (1990). We introduced into (2) also a dimensionless diameter of a wire and a force

$d=\sqrt{\frac{\pi H_{0}^{3}}{4 V_{m}}} \frac{\bar{d}}{H_{0}}, \quad p=\frac{4 \pi P H_{0}^{4}}{E V_{m}^{2}}$,

respectively, where $V_{m}$ is a volume of a spring material. The subscript 0 refers to the initial state (before loading) whereas the quantities without any subscript refer to the actual state under compression. The appropriate boundary conditions referring to a symmetrical mode of buckling, which leads to the first lower critical force, can be written as follows:

$w(0)=0, \quad w^{\prime}(1 / 2)=0$. 
The actual helix angle $\alpha$ can be evaluated from

$F_{1}=p-d^{4}\left(\frac{H_{0}}{R_{0}}\right)^{2} \frac{\cos ^{4} \alpha_{0}\left(\operatorname{tg} \alpha_{0}-\operatorname{tg} \alpha\right)\left(1+\nu+\operatorname{tg} \alpha_{0} \operatorname{tg} \alpha\right)}{\cos ^{3} \alpha\left(1+\nu+\operatorname{tg}^{2} \alpha\right)^{2}}=0$.

The ratio $R(\xi) / R_{0}=\rho(\xi)$ is described by

$F_{2}=\rho(\xi)-\frac{\cos ^{2} \alpha\left(1+\nu+\operatorname{tg}^{2} \alpha\right)}{\cos ^{2} \alpha_{0}\left(1+\nu+\operatorname{tg} \alpha_{0} \operatorname{tg} \alpha\right)}=0$

and (6) and (7) are discussed by Kruzelecki and Życzkowski (1990). The initial number of coils

$n_{0}=\varphi_{\max } / 2 \pi$,

$\varphi_{\max }=2 \int_{0}^{1 / 2} \frac{H_{0}}{R_{0}} \frac{\sqrt{\cos ^{2} \alpha_{0}-\left(\mathrm{d} R_{0} / H_{0} \mathrm{~d} \xi\right)^{2} \sin ^{2} \alpha_{0}}}{\sin \alpha_{0}} \mathrm{~d} \xi$,

as well as the actual number of coils

$n=\varphi_{\max } / 2 \pi$,

$\varphi_{\max }=2 \int_{0}^{1 / 2} \frac{\sqrt{\cos ^{2} \alpha-(\mathrm{d} r / \mathrm{d} \xi)^{2} \sin ^{2} \alpha_{0}}}{r \sin \alpha_{0}} \mathrm{~d} \xi$,

are obtained from the analysis of a geometry of a spring (under the assumption of symmetry of a spring) in an initial and actual configuration, respectively, and $\mathrm{d} r / \mathrm{d} \xi$ can be obtained from (7).

The following equality constraints are taken into account:

- volume of the spring material

$$
1=2 d^{2} \int_{0}^{1 / 2} \frac{\mathrm{d} \xi}{\sin \alpha_{0}}
$$

- compression rigidity of the spring

$$
\frac{1}{c_{0}}=\frac{8 \pi}{d^{4}} \int_{0}^{1 / 2}\left(\frac{R_{0}}{H_{0}}\right)^{2} \frac{1+\nu \cos ^{2} \alpha_{0}}{\sin \alpha_{0}} \mathrm{~d} \xi
$$

- slenderness ratio of the spring

$$
\frac{R_{0 c}^{2}}{H_{0}^{2}}=2 \int_{0}^{1 / 2}\left(\frac{R_{0}}{H_{0}}\right)^{2} \mathrm{~d} \xi
$$

where $c_{0}=\bar{c}_{0} H_{0}^{5} / E V_{m}^{2}$ is a dimensionless compression rigidity and $R_{0 c}$ is a radius of a reference spring. A reference spring with a constant radius $R_{0 c}$ and with a constant $\alpha_{0}$ is introduced to fix a comparative slenderness ratio and to calculate a corresponding critical force which can be compared with an optimal spring with a variable radius.

The second group contains so-called inequality constraints:

- strength condition

$$
\frac{\tau_{0}}{E} \geq \frac{p}{2}\left(\frac{V_{m}}{H_{0}^{3}}\right)^{1 / 2} r \frac{K(c, \alpha)}{\pi^{1 / 2} d^{3}}
$$

where

$$
K(c, \alpha)=1+\frac{5}{4 c}+\frac{7}{8 c^{2}}+\frac{\operatorname{tg}^{2} \alpha}{2}+\ldots,
$$

is a correction factor derived by Ancker and Goodier (1958) and Wahl (1963),

$c=\frac{2 r}{d} \sqrt{\frac{\pi H_{0}^{3}}{4 V_{m}}}$,

is a spring index and $\tau_{0}$ is an admissible shearing stress,

- closing up condition of neighbouring coils

$2 \pi r \operatorname{tg} \alpha \geq d \sqrt{\frac{4 V_{m}}{\pi H_{0}^{3}}}$,

- compatibility condition of geometry under compression

$\left|\frac{\mathrm{d} r}{\mathrm{~d} \xi}\right| \leq \frac{\cos \alpha}{\sin \alpha_{0}}$

- purely geometrical conditions in the initial state

$$
\begin{aligned}
& \alpha_{0 \ell} \leq \alpha_{0} \leq \alpha_{0 u}, \\
& R_{0 \ell} \leq R_{0} \leq R_{0 u},
\end{aligned}
$$

where subscripts $\ell$ and $u$ denote lower and upper values, respectively. When the constraint (13), (16) or (17) becomes the active condition it defines the lower value of a helix angle (admissible $\alpha_{0 \text { ad }}$ or closing $\alpha_{0 \text { cl }}$ or limit $\alpha_{0 \text { lt }}$, respectively) for optimization of $\alpha_{0}(\xi)$ and the upper value of a radius (admissible $R_{0 \text { ad }}$ or closing $R_{0 \mathrm{cl}}$ or limit $R_{0 \mathrm{lt}}$, respectively) for optimization of the radius of the spring. Then, using (6) and $(7)$, we can calculate the appropriate force $\left(p_{\mathrm{ad}}, p_{\mathrm{cl}}, p_{\mathrm{lt}}\right)$. These problems are discussed in detail by Kruzelecki (1995).

When optimizing a helical spring, two functional design variables can be considered: a function describing the initial helix angle $\alpha_{0}=\alpha_{0}(\xi)$ and a function describing the mean initial radius $R_{0}=R_{0}(\xi)$. Also the shape and size of the cross-section of a wire can be considered as a functional design variable, but we limit our investigation to the circular cross-section of constant diameter. The simplest variant of optimization, when both functions are prescribed is parametrical optimization. A prescribed function means that the type of function is assumed and it depends on some parameters which can be subjected to optimization.

We limit our investigations to the functions $\alpha_{0}\left(\xi, \beta_{i}^{\alpha}\right)$ and $R_{0}\left(\xi, \beta_{i}^{R}\right)$ depending, in general, on two parameters $(i=1,2)$, but only one of them is free and is subjected to optimization; the second one and the diameter of a wire can be evaluated from the equality constraints. Such a small number of parameters allows one, however, to show the nature of the optimization problems without using complicated and time-consuming special numerical procedures. We only consider optimization with respect to two parameters for some special cases.

We start from the simplest optimization problem, namely the optimization of one shape function (a helix angle or a radius) and finally we show the most general problem when both shape functions are sought.

\section{Optimization of $\alpha_{0}$ for prescribed $R_{0}$}

The optimization of a helix angle $\alpha_{0}(\xi)$ can be carried out for different prescribed $R_{0}(\xi)$. A prescribed $R_{0}$ means here a fixed value of the mean radius of a cylindrical spring, $R_{0}=$ constant. This gives the possibility to investigate the influence of a helix angle $\alpha_{0}$ on the critical force $p_{\mathrm{cr}}$. It also allows 
one to compare the results with those obtained for variational optimization (Kruzelecki 1995).

Two different types of functions describing $\alpha_{0}(\xi)$ are considered, namely piece-wise constant and smooth functions. Independently of the type and form of a function, the algorithm of calculations is always the same. First, a slenderness ratio of a spring is assumed, namely a value of $R_{0} / H_{0}$. Next, a compression rigidity $c_{0}$ and a dimensionless volume $v=V_{m} / H_{0}^{3}$ of a spring material are established. From two equality constraints (10) and (11) we can evaluate a diameter $d$ of a spring wire and one of free parameters. A remaining parameter must be assumed and then the shape function is uniquely defined. For such a set of parameters $\left[\alpha_{0}(\xi)\right.$ is already defined] the critical force $p_{\mathrm{cr}}$ is evaluated using (2). Also an admissible force $p_{\text {ad }}$, a closing up force $p_{\mathrm{cl}}$ and a limit force $p_{1 \mathrm{t}}$ can be evaluated from (13), (16) and (17), respectively. These forces $\left(p_{\mathrm{ad}}, p_{\mathrm{cl}}, p_{\mathrm{lt}}\right)$ can be compared with the critical force $p_{\mathrm{cr}}$ (which is maximized) and we are able to find which inequality constraint becomes an active condition. Such a procedure is repeated for different values of a parameter (parameters) until the extremum of the critical force $p_{\mathrm{cr}}$ is reached.

\subsection{A piece-wise constant $\alpha_{0}(\xi)$}

A piece-wise constant function describing an initial helix angle at half of the spring (symmetry of the spring) is assumed to be a two-sector function, namely:

$\alpha_{0}= \begin{cases}\alpha_{0 \ell}, & 0 \leq \xi \leq \xi_{0} \\ \alpha_{0 u}, & \xi_{0} \leq \xi \leq 1 / 2 .\end{cases}$

For the assumed constant value of $\xi_{0}$ we deal with one parameter whereas for a variable $\xi_{0}$ with a two-parameter optimization problem. A piece-wise constant $\alpha_{0}$ causes that, under compression, an actual helix angle $\alpha$ as well as the actual radius $R$ are also described by piece-wise constant functions. Hence, the compatibility condition of geometry under loading (17) cannot be satisfied in this case (we drop this constraint).

For a piece-wise constant $\alpha_{0},(2)$ can be integrated analytically in two intervals, namely $\left(0, \xi_{0}\right)$ and $\left(\xi_{0}, 1 / 2\right)$. Utilizing the boundary conditions and the continuity conditions we obtain

$\frac{k_{2} \gamma_{1}}{k_{1} \gamma_{2}} \operatorname{tg} k_{1} \frac{\psi_{1}}{2}=\operatorname{ctg} k_{2} \frac{\psi_{2}}{2}$,

where

$\gamma=\frac{\sin \alpha}{\sin \alpha_{0}}+\frac{p}{d^{4}} \frac{1+\nu \sin ^{2} \alpha}{\sin \alpha_{0}} \frac{r^{2}}{2 \mu_{s}}$,

$k^{2}=\frac{p}{2 d^{4}} \frac{2+\nu \cos ^{2} \alpha}{\sin \alpha_{0}} \gamma, \quad \psi_{1}=2 \xi_{0}, \quad \psi_{2}=1-\psi_{1}$.

In (22) and (23) the subscripts 1 and 2, distinguishing sectors, are neglected. The actual number of coils, which influences $\mu_{s}$, can be evaluated from

$n=\left(\frac{H_{0}}{R_{1}} \frac{\psi_{1}}{\operatorname{tg} \alpha_{1}} \frac{\sin \alpha_{1}}{\sin \alpha_{01}}+\frac{H_{0}}{R_{2}} \frac{\psi_{2}}{\operatorname{tg} \alpha_{2}} \frac{\sin \alpha_{2}}{\sin \alpha_{02}}\right) / 2 \pi$.

Additionally, the condition of equality of compression forces in both sectors of the spring leads to

$\frac{\cos ^{4} \alpha_{01}}{\cos ^{3} \alpha_{1}} \frac{\left(\operatorname{tg} \alpha_{01}-\operatorname{tg} \alpha_{1}\right)\left(1+\nu+\operatorname{tg} \alpha_{01} \operatorname{tg} \alpha_{1}\right)}{\left(1+\nu+\operatorname{tg}^{2} \alpha_{1}\right)^{2}}=$ $\frac{\cos ^{4} \alpha_{02}}{\cos ^{3} \alpha_{2}} \frac{\left(\operatorname{tg} \alpha_{02}-\operatorname{tg} \alpha_{2}\right)\left(1+\nu+\operatorname{tg} \alpha_{02} \operatorname{tg} \alpha_{2}\right)}{\left(1+\nu+\operatorname{tg}^{2} \alpha_{2}\right)^{2}}$.

Using (21) and (25) together with (6) and (7), written for each sector of the spring, we can find the critical force $p_{\mathbf{c r}}$. Parameters which are subjected to optimization can be a helix angle $\alpha_{01}$ and a coordinate $\xi_{0}$, expressed here by the parameter $\psi_{1}$. A helix angle $\alpha_{02}$ in the middle sector of a spring and a diameter of a wire are defined by

$c_{0}=\frac{d^{4}}{4 \pi}\left(\frac{H_{0}}{R_{0}}\right)^{2}$.

$\left[\frac{\sin \alpha_{01} \sin \alpha_{02}}{\psi_{1}\left(1+\nu \cos ^{2} \alpha_{01}\right) \sin \alpha_{02}+\psi_{2}\left(1+\nu \cos ^{2} \alpha_{02}\right) \sin \alpha_{01}}\right]$,

$\frac{1}{d^{2}}=\frac{\psi_{1}}{\sin \alpha_{01}}+\frac{\psi_{2}}{\sin \alpha_{02}}$,

obtained from the equality constraints (11) and (10), respectively.

First of all, a simpler problem of one-parameter optimization is investigated. It is assumed that a coordinate $\xi_{0}$ is fixed, namely $\xi_{0}=0.25$ (one half of the spring is divided into two segments of equal length) and a helix angle $\alpha_{01}$ is subjected to optimization. Calculations are performed for compression rigidity $c_{0}=0.1$ and for different slenderness ratios expressed by $R_{0} / H_{0}$. The results are presented in Fig. 1. There are two different types of $p_{\mathrm{cr}}$ curves. First, for springs with large slenderness ratios (for $R_{0} / H_{0}<0.1065$ ), each curve has a local maximum referring to the optimal solution. It is worth stressing, that a local maximum is obtained, in general, for a rather large difference between $\alpha_{02}$ and $\alpha_{01}$ $\left(\alpha_{01}<\alpha_{02}\right)$, so the number of coils in the middle sector of a spring is rather small. For springs with smaller slenderness ratios $\left(R_{0} / H_{0}>0.1075\right)$ the curves have return points. This means, that such a spring can become one which is always stable. The exact value of a parameter $R_{0} / H_{0}$ for which the curve changes its character is not found. It can be expected that for $0.1065 \leq R_{0} / H_{0} \leq 0.1075$ there is a spring for which both forms are equally possible.

The curves describing the closing up condition are plotted in Fig. 1. The upper curve refers to the case when a finite diameter of a wire is not taken into account in the closing up condition of neighbouring coils $[\alpha(0)=0$ the weakest condition], whereas the lower curve is obtained for the assumed dimensionless volume of a spring material $v=0.005$. For this $v$ the closing up constraint becomes an active condition even for springs with large slenderness ratio. Then, the optimal solution is obtained for $p_{\mathrm{cr}}=p_{\mathrm{cl}}$ - the intersection of the curves. For springs with a smaller slenderness ratio, for which the return point occurs, the maximal (optimal) force is defined by the closing up condition.

The profit obtained for the optimal piece-wise constant $\alpha_{0}$, in the entire domain of $R_{0} / H_{0}$, is very high; it is even over $100 \%$ (the increment of the critical force).

The influence of $\xi_{0}\left(\xi_{0}\right.$ - additional free parameter) on the critical force is also investigated. A value of $\xi_{0} \neq 0.25$ gives the possibility to obtain a more profitable distribution of the actual bending rigidity and it can lead to a higher critical force. In Fig. 1 the appropriate curves are marked 


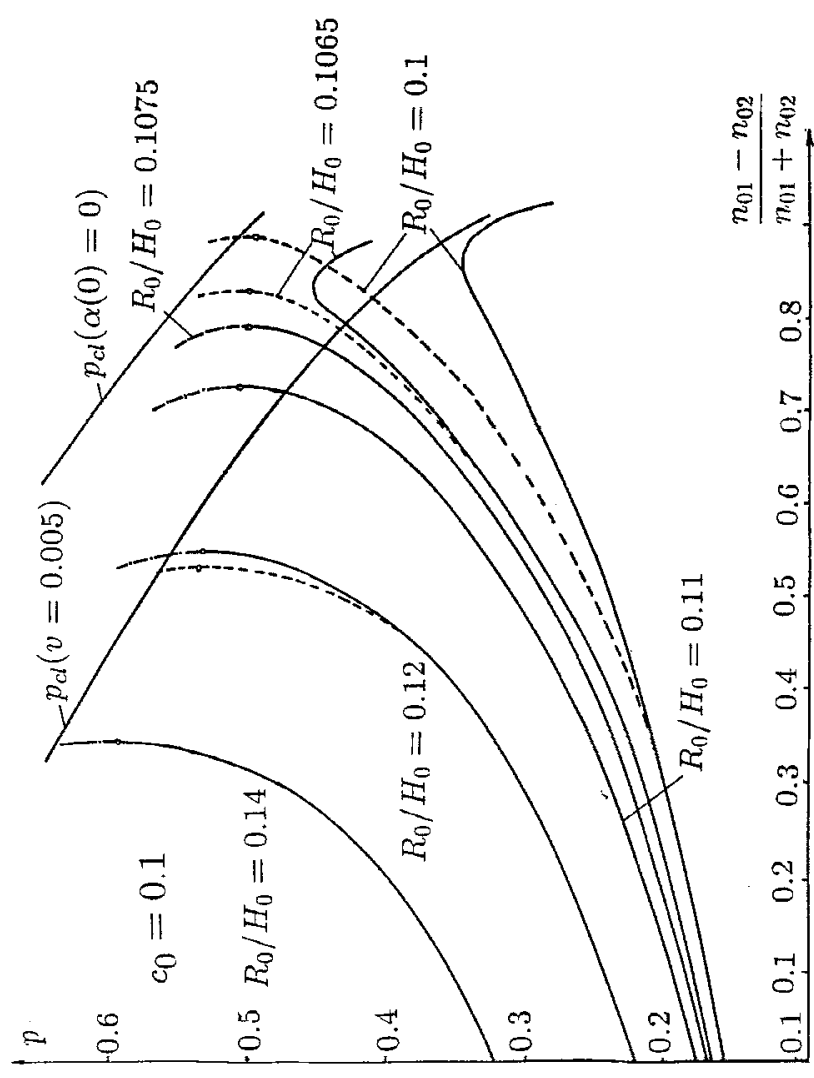

Fig. 1. Results for piece-wise constant $\alpha_{0}$ springs

by dashed lines. For springs with a smaller slenderness ratio and with $\xi_{0} \neq 0.25$, the return points "move" to the left, but these changes are rather small. Consequently, the results are not changed qualitatively and the quantitative changes are small. For springs with a larger slenderness ratio, for which the maximum of $p_{c r}$ is obtained, it is possible now to obtain the return points. Therefore, the additional parameter $\xi_{0}$ can change the results qualitatively as well as quantitatively. For small $R_{0} / H_{0}$ a helix angle $\alpha_{02}$ becomes large in the middle sector of a spring and its value should be bounded by the appropriate geometrical constraint (18).

In Fig. 1, the curves connected with the strength constraint are not shown. It refers to a high value of the admissible stress $\tau_{0}$. The lower admissible stress leads to activity of the strength condition and the optimal solution can be obtained at the intersection of the appropriate curves.

We are able to compare the results obtained from the parametrical optimization with those from the variational optimization (Krużelecki 1995). As an example we take a spring with $R_{0} / H_{0}=0.075$ and with the following geometrical constraints: $0.1 \leq \alpha_{0} \leq \pi / 4$. These constraints are the only active conditions in this case ( $\alpha_{0}$ jumps from 0.1 to $\pi / 4$ ). The coordinate $\xi_{0}=0.1974$ results from (12) and it is in very good agreement with the variational solution. The critical force $p_{\mathrm{cr}}=0.1887$ is slightly smaller (about $1 \%$ ) than that obtained by Kruzielecki (1995).

\subsection{Helix angle $\alpha_{0}(\xi)$ described by continuous functions}

A general form of these functions can be written as follows:

$\alpha_{0}(\xi)=\beta_{0}+\beta f(\xi)$, where $f(\xi)$ is a certain prescribed function, $\beta$ is a design parameter and $\beta_{0}$ can be evaluated from the equality constraint (one parameter optimization). Some examples of $f(\xi)$ considered in this paper are shown in Table 1. In Table 1 the boundary conditions, which $f(\xi)$ and consequently $\alpha_{0}(\xi)$ satisfies, are also shown.

Table 1. Examples of $f(\xi)$

\begin{tabular}{|c|l|l|}
\hline No. & $f(\xi)$ & Boundary conditions for $f(\xi)$ \\
\hline \hline 1 & $\xi$ & - \\
\hline 2 & $\xi(1-\xi)$ & $f^{\prime}(1 / / 2)$ \\
\hline 3 & $\xi^{2}(1-4 / / 3 \xi)$ & $f^{\prime}(0)=f^{\prime}(1 / / 2)=0$ \\
\hline 4 & $\xi^{2}\left(1-8 / / 3 \xi+2 \xi^{2}\right)$ & $f^{\prime}(0)=f^{\prime}(1 / / 2)=f^{\prime \prime}(0)=0$ \\
\hline 5 & $\xi^{3}\left(1-3 \xi+12 / / 5 \xi^{2}\right)$ & $f^{\prime}(0)=f^{\prime}(1 / / 2)=f^{\prime \prime}(0)=f^{\prime \prime}(1 / / 2)=0$ \\
\hline
\end{tabular}

As an example, we consider the simplest linear function describing an initial helix angle $\alpha_{0}(\xi)$ (No. 1, Table 1). Calculations are performed for the compression rigidity $c_{0}=0.1$ and for different values of a parameter $R_{0} / H_{0}$. The results are presented in Fig. 2. Three different families of curves are shown there. The first one (thick lines) represents the dependence of a critical force $p_{\mathrm{cr}}$ on a parameter $\beta$ for different $R_{0} / H_{0}$. For $\beta>0$ and the assumed value of $R_{0} / H_{0}$ the critical force always increases, but the curves do not show a local maximum. For springs with a small slenderness ratio the curves $p_{\mathrm{cr}}(\beta)$ have return points, e.g. $R_{0} / H_{0}=0.185$. The thin lines refer to the closing up condition for different values of a dimensionless volume $v$ of a spring material and they limit critical forces. The most outside line is connected with the weakest form of this constraint, namely $\alpha(0)=0$. The other curves of this type refer to larger values of $v$. The points of intersection of $p_{\mathrm{cr}}(\beta)$ and $p_{\mathrm{cl}}(\beta)$ define the optimal solutions when the remaining constraints are passive.

The third type of curves, $p_{\text {ad }}(\beta)$, results from the strength condition, which can be satisfied in the form of an equality in the point where the actual helix angle $\alpha$ is the largest (in this point the actual radius $R$ is also the largest). In Fig. 2 a few such curves are shown for different values of $\tau_{0} / E$ and $v$. Increasing $\alpha_{0}$ causes a small decrease of the admissible force $p_{\text {add }}$. Hence, the lowest point of intersection of a pair of curves defines the optimal solution for passive other constraints. Increasing $p_{\text {ad }}$ is possible only for better materials a higher value of $\tau_{0} / E$. Also the optimization of the shape of a cross-section of a wire can improve the admissible $p_{\text {ad }}$ and consequently the optimal solution (Eschenauer and Kobelev 1991; Kamiya and Kita 1990).

A higher $p_{\mathrm{cl}}$ can be obtained using an appropriate distribution of $\alpha_{0}(\xi)$. The closing up of neighbouring coils takes place in the region where a helix angle is the smallest (a distance between neighbouring coils is the smallest); this means in the region closest to the supports. It seems that a better solution can be obtained using a more uniform distribution of $\alpha(\xi)$ in this region. This leads to a larger distance between the first and second coils and can be done by taking appropriate $f(\xi)$. Hence, the assumed form No. 2 in Table 1 of $f(\xi)$ which satisfies the condition $\alpha^{\prime}(0)=0$.

A higher value of the critical force $p_{\text {cr }}$ can be obtained for more profitable distribution of $\alpha_{0}$. Particularly, it is important in the region of the middle of a spring. In this region, for a possible long sector, we should apply a possible large value of $\alpha_{0}$. The proposed functions $f(\xi)$ Nos. 3 and 4 in Table 1 


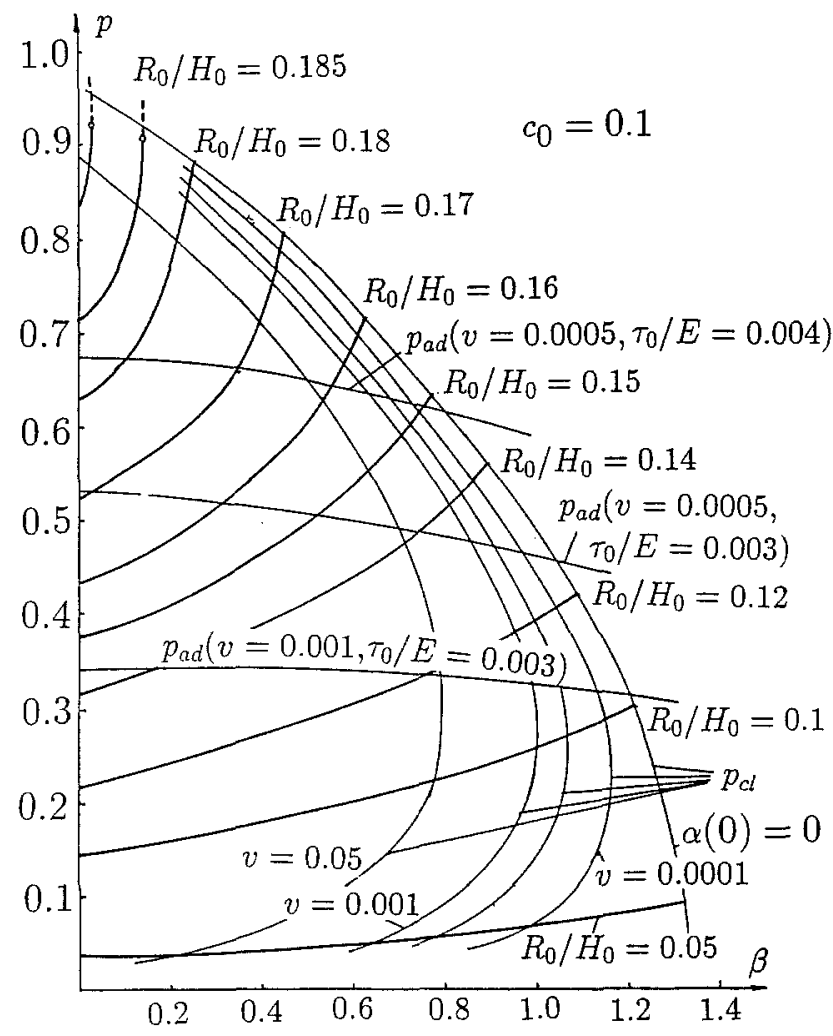

Fig. 2. Results for springs with linear $\alpha_{0}(\xi)$; Table 1, No. 1

are chosen in this way. Using $f(\xi)$ Nos. 3 and 4 we obtain an increment of $p_{\text {cr }}$ which should be followed by an increment of $p_{\mathrm{cl}}$ because the optimal solution is reached at the intersection of these two curves. The proposed function No. 5 in Table 1 satisfies those requirements but the results for this function and for the other $f(\xi)$ are not presented here. In Fig. 3 the optimal solutions $\left(p_{\mathrm{cr}}=p_{\mathrm{cl}}\right)$ obtained for the weakest active closing up constraint $[\alpha(0)=0]$ and for some of $f(\xi)$ presented in Table 1 are shown. A tendency of increasing of $p_{\text {cr }}$ for each improved function $f(\xi)$, i.e. for improved $\alpha_{0}(\xi)$, can be observed. For comparison, the results for springs with a constant helix angle $\alpha_{0}$ are shown (the lowest curve), for which $p_{\mathrm{cr}}<p_{\mathrm{cl}}$.

\section{Optimization of $R_{0}$ for prescribed $\alpha_{0}$}

Optimization of the mean radius $R_{0}$ of a spring is possible for different functions describing $\alpha_{0}(\xi)$. We assume that prescribed $\alpha_{0}$ means $\alpha_{0}(\xi)=$ constant. It allows us to find a profit due to optimization of the radius only.

We limited our investigation to the simplest one parameter optimization problems describing a variable radius of a spring $R_{0}(\xi)$ by different functions, namely by piece-wise constant as well as continuous functions which contain two parameters only. Independently of the type of function the procedure of calculations is always the same. We assume a slenderness ratio of a reference spring taking a parameter $R_{0 c} / H_{0}$. Next, from the equality constraints (10), (11) and (12) we can calculate the diameter of a spring wire, a constant helix angle $\alpha_{0}$ and one of the parameters characterizing the radius of a spring $R_{0}(\xi)$. A diameter of a wire $d$ and a

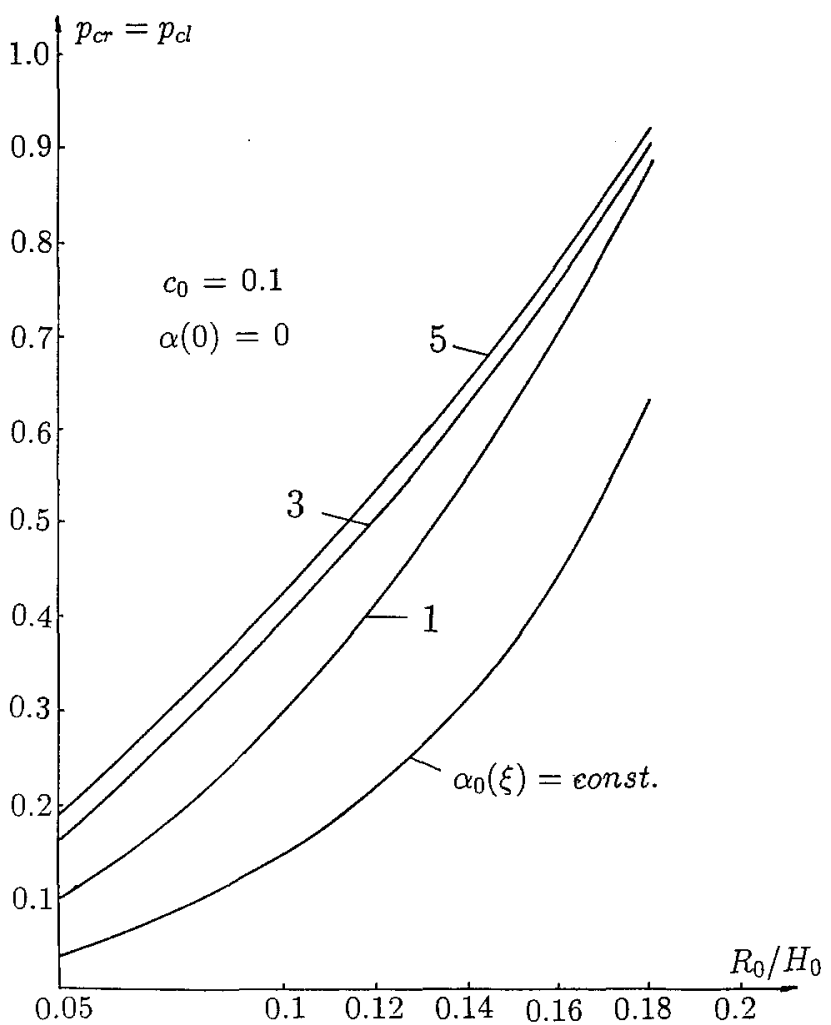

Fig. 3. Optimal solutions connected with the active closing up constraints; 1, 3, 5 denote the number of functions in Table 1

helix angle $\alpha_{0}$ obtained for an optimal spring are the same as for the reference spring. For this set of parameters we calculate a critical force $p_{\mathrm{cr}}$ using (2) and (6) and (7). We are able also to find an admissble force $p_{\text {ad }}$, a closing up force $p_{\text {cl }}$ and a limit force $p_{\mathrm{lt}}$ from the constraints (13), (16) and (17), respectively. This gives us the possibility, at each calculation step, to find which constraint becomes active, i.e. to find the boundaries of the admissible region.

\subsection{A piece-wise constant $R_{0}(\xi)$}

We assume that the radius can be described by a function

$R_{0}= \begin{cases}R_{01}, & 0 \leq \xi \leq \xi_{0} \\ R_{02}, & \xi_{0} \leq \xi \leq 1 / 2\end{cases}$

where $\xi_{0}$ is a coordinate of the division of half of a spring into two sectors with constant initial radii $R_{01}$ and $R_{02}$. This type of $R_{0}(\xi)$ was obtained for variational optimization (Kruzelecki 1995). Because of a jump-like $R_{0}(\xi)$ the compatibility condition of geometry is not satisfied in the point $\xi_{0}$.

In (29) there are three parameters, namely $R_{01}, R_{02}$ and $\xi_{0}$. So, in the general case, we have a two-parameter optimization problem. The algorithm of calculations and the equations describing $p_{\mathrm{Cr}}$ can be adopted from the optimization of a piece-wise constant helix angle. It is necessary in (22) and (23) to apply subscripts 1 and 2 (to distinguish sectors) for $R, R_{0}$ and $\alpha$, leaving $\alpha_{0}$ without any subscript because an initial helix angle is common for both sectors. Then, (25) should be rewritten in the form 
$\frac{H_{0}^{2}}{R_{01}^{2}} \frac{\left(\operatorname{tg} \alpha_{0}-\operatorname{tg} \alpha_{1}\right)\left(1+\nu+\operatorname{tg} \alpha_{0} \operatorname{tg} \alpha_{1}\right)}{\cos ^{3} \alpha_{1}\left(1+\nu+\operatorname{tg}^{2} \alpha_{1}\right)^{2}}=$

$\frac{H_{0}^{2}}{R_{02}^{2}} \frac{\left(\operatorname{tg} \alpha_{0}-\operatorname{tg} \alpha_{2}\right)\left(1+\nu+\operatorname{tg} \alpha_{0} \operatorname{tg} \alpha_{2}\right)}{\cos ^{3} \alpha_{2}\left(1+\nu+\operatorname{tg}^{2} \alpha_{2}\right)^{2}}$,

whereas (26) and (27) lead to

$c_{0}=\frac{d^{4}}{4 \pi} \frac{\sin \alpha_{0}}{\left(\psi_{1} R_{01}^{2} / H_{0}^{2}+\psi_{2} R_{02}^{2} / H_{0}^{2}\right)\left(1+\nu \cos ^{2} \alpha_{0}\right)}$,

$\frac{1}{d^{2}}=\frac{\psi_{1}+\psi_{2}}{\sin \alpha_{0}}$

respectively.

Calculations are performed for $c_{0}=0.1$ and for different values of $R_{0 c} / H_{0}$. A one-parameter optimization problem, assuming $\psi_{1}=0.5\left(\xi_{0}=0.25\right)$, as well as a two-parameter optimization problem ( $\psi_{1}$ - free parameter) are considered. Profits of the optimization, measured by the relative increment of the critical force $p_{c r}$, in both cases are rather small in comparison with the optimization of $\alpha_{0}$.

For one-parameter optimization, when the inequality constraints are passive, the optimal solution leads to a spring with a radius tending to zero in the middle part of the spring, $R_{02} \rightarrow 0$. However, taking even a very small lower bound $R_{0 \ell}$ may reduce this small profit radically. Some results are presented in Table 2.

For two-parameter optimization, the lack of geometrical constraints leads to a degenerated spring; for almost the entire spring $R_{02} \rightarrow 0$ and $R_{01}$ takes such a large value that a compression rigidity equals the assumed value of $c_{0}$.

In the middle sector of a spring the bending rigidity should be as high as possible. This leads to a possible small radius in this sector, $R_{02}=R_{0 \ell}$. The most profitable distribution of bending rigidity is that, for which a sector with $R_{02}=R_{0 \ell}$ is possibly long. Then, because of the assumed value of compression rigidity $c_{0}$ in the remaining sector, we obtain $R_{01}=R_{0 u}$ and a coordinate $\xi_{0}$ results from (11).

Calculations are performed for $R_{0 c} / H_{0}=0.075,0.1,0.12$. The results (Table 2) are the same as those obtained for variational optimization (Krużelecki 1995).

\subsection{Radius $R_{0}(\xi)$ described by continuous functions}

Besides cylindrical springs with constant helix angle, different shaped springs are often used, namely conical and parabolical springs whose shapes can be described by

$\frac{R_{0}(\xi)}{H_{0}}=\frac{r_{0}}{H_{0}}+\beta f\left(\xi, \xi_{0}\right)$

where $\beta$ is a parameter subjected to optimization $(\beta<0$ leads to a concave spring), and $r_{0}$ is the unknown radius in a fixed point $\xi_{0}$. For a constant helix angle a variable pitch is obtained. The distance between neighbouring coils in the sectors near supports of a spring is larger than in the middle sector. Hence, the actual compression and bending rigidities are larger in the middle part of the spring than in regions of support (Krużelecki and Życzkowski 1990), but a bending rigidity changes very slightly. So, it can be expected that the profit of optimization is rather small. Both, a closing up constraint and strength constraint become active conditions for $\xi=0$; the first one due to the largest compliance in this region, the latter one with respect to the highest bending moment acting on a wire in this region.

First of all, calculations are performed for a conical spring

$f\left(\xi, \xi_{0}\right)=\xi-\xi_{0}$,

and for a spring

$f\left(\xi, \xi_{0}\right)=\xi^{3}-\xi_{0}^{3}-3\left(\xi^{4}-\xi_{0}^{4}\right)+\frac{12}{5}\left(\xi^{5}-\xi_{0}^{5}\right)$

which we call here a parabolical spring although it does not describe a classical parabolic curve, where $\xi_{0}=0.25$ is assumed in both cases. It turns out that neither the strength constraint nor the closing up constraint are active conditions for these types of springs. The active constraint becomes a geometrical condition limiting a lower bound of a radius. In the limit case a radius in the middle of the spring tends to zero, $R_{0}(1 / 2) \rightarrow 0$, which is the possible weakest limitation of the admissible region. The results for $c_{0}=0.1$ are presented in Table 2 . The profits of optimization are very small in both cases; slightly better are solutions connected with a parabolic spring because this shape is closer to a piece-wise constant radius spring.

Table 2. Results of optimization for springs of various shape

\begin{tabular}{|l|c|c|c|c|c|}
\hline$\frac{R_{0 c}}{H_{0}}$ & \multicolumn{5}{|c|}{$p_{\text {cr }}$} \\
\cline { 2 - 6 } & cylindrical & conical & parabolical & hyperbolical & $\begin{array}{c}\text { piece-wise } \\
\text { constant }\end{array}$ \\
\hline 0.075 & 0.0816 & 0.0839 & 0.0840 & 0.0839 & 0.0842 \\
\hline 0.10 & 0.1495 & 0.1588 & 0.1595 & 0.1589 & 0.1600 \\
\hline 0.12 & 0.2250 & 0.2487 & 0.2510 & 0.2501 & 0.2541 \\
\hline
\end{tabular}

Additionally, we consider a hyperbolical spring described by

$\frac{R_{0}(\xi)}{H_{0}}=\left\{\frac{r_{0}^{2}}{H_{0}^{2}}-\beta\left[\left(\frac{1}{2}-\xi\right)^{2}-\left(\frac{1}{2}-\xi_{0}\right)^{2}\right]\right\}^{\frac{1}{2}}$,

whose shape is close to the so-called upholsterer's spring, where $\beta<0$ and $\xi_{0}=1 / 2(1-1 / \sqrt{3})$ leads to $r_{0}=R_{0 c}$. The results of optimization are shown in Table 2 .

5 Optimization of springs for prescribed function $F\left(R_{0}, \alpha_{0}\right)$ - springs with a constant initial pitch

Generally, the shape of a spring $R_{0}(\xi)$ and a helix angle $\alpha(\xi)$ can be chosen arbitrarily. However, there is a large group of springs, namely springs with a constant pitch, for which an exact relation between these two quantities exists:

$h_{0}=[\xi(\varphi+2 \pi)-\xi(\varphi)]=$ constant,

or taking into account that $\mathrm{d} \xi / \mathrm{d} \varphi=$ constant we obtain

$\xi=\frac{\varphi}{2 \pi} \frac{h_{0}}{H_{0}}$

where $\varphi$ denotes a polar angle, $h_{0}$ is an initial pitch of the spring. In the general case, an initial helix angle $\alpha_{0}$ can be described by

$\frac{1}{\sin _{0}(\xi)}=\sqrt{\left(\frac{R_{0}}{H_{0}}\right)^{2}\left(\frac{\mathrm{d} \varphi}{\mathrm{d} \xi}\right)^{2}+\left(\frac{\mathrm{d} R_{0}}{H_{0} \mathrm{~d} \xi}\right)^{2}+1}$,

where $\mathrm{d} \varphi / \mathrm{d} \xi=2 \pi H_{0} / h_{0}$ results from (38). This represents relation $F\left(R_{0}, \alpha_{0}\right)$; a change of $R_{0}$ causes an appropriate change of the helix angle $\alpha_{0}$ in the same region. 
The optimization of a spring with a constant pitch, in general, can be done in two different ways. The first is designing a radius $R_{0}(\xi)$; a variable helix angle $\alpha_{0}(\xi)$ then results from (39). The second way is connected with assuming $\alpha_{0}(\xi)$ and finding $R_{0}(\xi)$. This way is more complicated because it is necessary to solve a nonlinear first-order differential equation [which is obtained from (39)] for each change of $\alpha_{0}$. In this paper the first way is used. To compare the results we apply the same functions describing $R_{0}(\xi)$ as for the optimization of the radius for constant $\alpha_{0}$, namely conical, parabolical and hyperbolical springs, and springs with a piece-wise constant radius.

Independent of the shape of a spring, qualitatively the same results are obtained (some differences are observed for a piece-wise constant radius spring). For a larger ratio of $R_{0 c} / H_{0}$ the critical force $p_{\mathrm{Cr}}$ is limited by a closing up condition [we use here $\alpha(0)=0$ ]. Then, we obtain $R_{0}(1 / 2) \neq 0$ and $\alpha_{0}(1 / 2)$ is reasonably large. For smaller parameters, e.g. $R_{0 c} / H_{0}=0.075$, the critical force results from a condition: $R_{0}(0) \rightarrow 0$, but simultaneously $\alpha_{0}(1 / 2) \rightarrow \pi / 2$. For a spring with a piece-wise constant radius, for larger values of $R_{0} / H_{0}$, such a distribution of $R_{0}(\xi)$ and $\alpha_{0}(\xi)$ is obtained that an optimal spring always becomes stable (return point occurs). For smaller $R_{0} / H_{0}$ the maximal critical force is obtained from the condition $R_{0}(1 / 2) \rightarrow 0$.

Calculations are performed for $c_{0}=0.1$ and for three chosen slenderness ratios: $R_{0} / H_{0}=0.075,0.1,0.12$. The results, for a parabolic spring only, are presented in Fig. 4. For comparison, the results of the optimization of the radius only $\left(\alpha_{0}=\right.$ constant) are also shown. The profit of optimization is relatively high. This is due to a profitable distribution of $\alpha_{0}(\xi)$; the influence of $R_{0}(\xi)$ on the critical force is rather small. This can be seen from comparison with results obtained for optimization of the radius only.

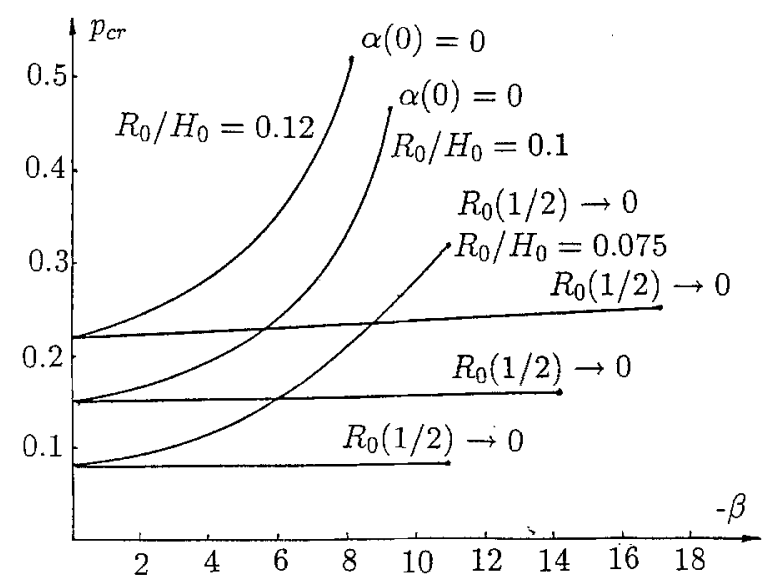

Fig. 4. Results for parabolic springs; upper curves - constant pitch springs, lower curves - springs with constant $\alpha_{0}$

\section{Optimization of $R_{0}$ and $\alpha_{0}$ as independent func- tions}

This is the most general optimization problem considered in this paper. Each function describing a geometry of a spring contains, for example, one free parameter only. This means that we are dealing with two-parameter optimization (the lowest possible number of parameters).

Both functions can be chosen arbitrarily, but we make capital of previously considered optimization problems. It is decided that optimal solutions are sought among parabolical springs defined by (33) and (35) and a helix angle can be described by

$\alpha_{0}(\xi)=\beta_{0}+\alpha_{0 h}\left(\xi, \beta_{R}\right)+\beta \xi^{3}\left(1-3 \xi+\frac{12}{5} \xi^{2}\right)$.

The first term in (40), $\beta_{0}$, can be evaluated from a condition of given compression rigidity. The second one, which refers to a spring with constant pitch $h_{0}$, depends on the shape of the spring and is described by (39), where $\beta_{R}$ is a parameter in a shape function $R_{0}(\xi)$. The last term is a parabolic distribution of a helix angle analysed previously; $\beta$ is a free parameter subjected to optimization. We use the above distribution of $\alpha_{0}$ because both basic terms in (40) lead to a very good improvement of the performance of the spring when each function is considered separately.

It is assumed that a strength constraint remains a passive condition (a high value of $\tau_{0} / E$ ) and additionally that geometrical constraints have the weakest form, namely: $R_{0 \ell} \rightarrow 0$ and $\alpha_{0 u} \rightarrow \pi / 2$. Moreover, a closing up constraint takes the form $\alpha(0)=0$. This allows one to find optimal solutions even for extreme variants of the geometry of the spring. The results for $c_{0}=0.1$ are presented in Fig. 5, where the critical force $p_{\mathrm{Cr}}$ is plotted versus $R_{0 c} / H_{0}$ for the optimal solutions; three intersected curves are shown. For large slenderness ratios (small $R_{0 c} / H_{0}$ ), when $R_{0 c} / H_{0} \leq 0.065$, the optimal solution refers to a case when a critical force $p_{\mathrm{cr}}$, a closing up force $p_{\mathrm{cl}}$ and a limit force $p_{\mathrm{It}}$ are equal, $p_{\mathrm{cr}}=p_{\mathrm{cl}}=p_{\mathrm{It}}$. Such optimal springs are "concave" (smaller radius in the middle part of the spring). Next, for $0.065 \leq R_{0 c} / H_{0} \leq 0.1075$, the optimal solution is obtained when $p_{\mathrm{cr}}=p_{\mathrm{cl}}$. For such a spring radius in the middle of the spring tends to zero, $R_{0}(1 / 2) \rightarrow 0$. For $R_{0 c} / H_{0} \geq 0.1075$, as an optimal solution we obtain such a spring for which $p_{\mathrm{cr}}=p_{\mathrm{cl}}$ and simultaneously the critical force $p_{\text {cr }}$ reaches its local maximum. In this case, for $R_{0 c} / H_{0} \leq 0.12$, we have a concave spring whereas for $R_{0 c} / H_{0} \geq 0.12$ an optimal spring is convex. For all investigated $R_{0 c} / H_{0}$, the closing up constraint in the weakest form is always an active condition, whereas other constraints become active conditions interchangeably.

The optimal solutions for cylindrical springs with a parabolical and conical distribution of the helix angle, as well as for springs with constant $\alpha_{0}$ are shown in Fig. 4. The profit obtained from the optimization of both functions, in comparison to a parabolic distribution of $\alpha_{0}(\xi)$, is not too high and it depends on $R_{0 c} / H_{0}$. The smallest profit is connected with small slenderness ratios (large $R_{0 c} / H_{0}$ ) and the largest increment of a critical force refers to springs with mean values of $R_{0 c} / H_{0}$.

All results presented here are connected with $c_{0}=0.1$. The assumption of a different value of $c_{0}$ may lead, first of all, to quantitatively different results. The qualitative changes can be connected with a different order of activity of the inequality constraints. 


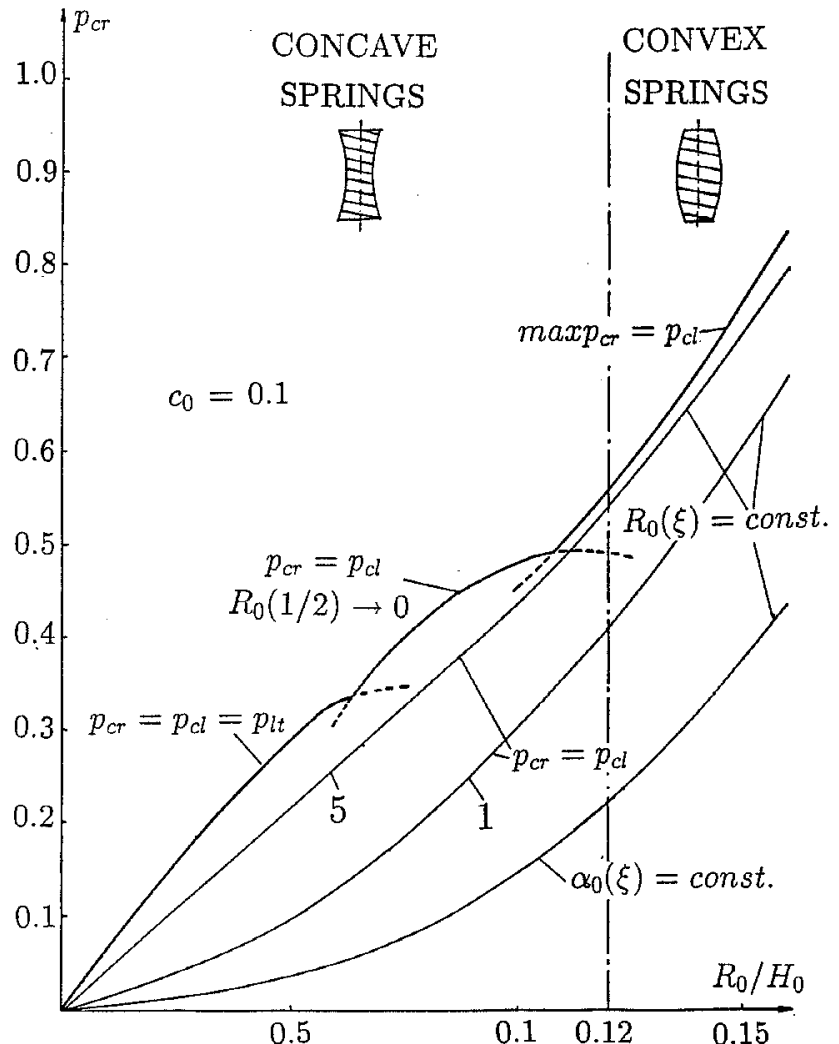

Fig. 5. Results for optimization of $\alpha_{0}$ and $R_{0}$ as independent functions; 1,5 denote the number of functions in Table 1

\section{Conclusions}

In this paper several problems of the parametrical optimization of compression helical springs are considered, namely: optimization of a helix angle for prescribed radius, optimization of a radius for prescribed helix angle, optimization of springs with constant pitch, and optimization of both independent shape functions. It turns out that the main profit of optimization is connected with the optimal distribution of the helix angle; an optimal spring has smaller $\alpha_{0}$ in regions of support and larger $\alpha_{0}$ in the middle region of a spring. Calculations show that it is possible to find a distribution of the helix angle such that the spring does not lose its stability under any axial force (an always stable spring). Such a spring is easier to obtain for a smaller slenderness ratio.

The influence of $R_{0}$ on the critical force is much lower. Usually, the shape of such an optimal spring is concave; a small $R_{0}$ is obtained in the middle region of a spring and a larger one in the regions of supports. The typical improvements are a few percent only, whereas that obtained by optimization of $\alpha_{0}$ can be even over $100 \%$.
The results show that optimization of $\alpha_{0}$ and $R_{0}$ as independent functions leads to a very small improvement in comparison with optimization of the helix angle only. The shape of a spring with a variable radius can be complicated and more expensive to manufacture. Hence, application of cylindrical springs with the optimal distribution of the helix angle only seems to be adequate.

The highest profit is obtained for springs with a piecewise constant helix angle and the results are close to those obtained for variational optimization (Krużelecki 1995).

\section{Acknowledgement}

This paper was partly supported by Grant PB 0269/P4/93/05.

\section{References}

Ancker, G.K.; Goodier, J.N. 1958: Theory of pitch and curvature correction for the helical spring. J. Appl. Mech. 25, 466-495

Eschenauer, H.A.; Kobelev, V.V. 1991: Shape anisotropy optimization of helical springs. Proc. 1st European Solid Mechanics Conf. (held in Munich)

Henderson, G.R. 1884-1885: Graphical method of designing springs. Trans. ASME 16, 92-96

Kamiya, N.; Kita, E. 1990: Boundary element method for quasiharmonic differential equation with application to stress analysis and shape optimization of helical springs. Comp. \& Struct. 37, 81-86

Krużelecki, J. 1990: Optimal design of helical springs. Mech. Teor. Stos. 28, 109-114

Krużelecki, J. 1993: On general helical springs optimized against instability. In: Herskovits, J. (ed.) Structural optimization '93, Volume 2, pp. 19-26. Rio de Janeiro: COPPE

Krużelecki, J. 1995: Variational optimization of compression helical springs with respect to their stability. Eng. Opt. 24, 293-317

Krużelecki, J. 1996: Unimodal and bimodal optimization of compression helical springs against instability. In: Olhoff, N.; Rozvany, G.I.N. (eds.) WCSMO-1, First World Congress of Structural and Multidisciplinary Optimization (held in Goslar, Germany, 1995), pp. 683-688. Oxford: Pergamon

Krużelecki, J.; Życzkowski, M. 1990: On the concept of an equivalent column in the problem of stability of compressed helical springs. Ing.-Arch. 60, 367-377

Wahl, A.M. 1963: Mechanical springs. New York: McGraw Hill

Ziegler, H. 1982: Arguments for and against Engeasser's formulas. Ing.-Arch. 52, 105-113 